\title{
Montessori et les enfants nomades : forme scolaire et mouvement de l'enfant
}

\section{Bérengère Kolly}

\section{(2) OpenEdition \\ Journals}

Édition électronique

URL : http://journals.openedition.org/trema/4309

DOI : $10.4000 /$ trema.4309

ISSN : 2107-0997

Éditeur

Faculté d'Éducation de l'université de Montpellier

Édition imprimée

Date de publication : 1 novembre 2018

ISSN : 1167-315X

Référence électronique

Bérengère Kolly, «Montessori et les enfants nomades : forme scolaire et mouvement de l'enfant », Tréma [En ligne], 50 | 2018, mis en ligne le 01 novembre 2018, consulté le 19 avril 2019. URL : http:// journals.openedition.org/trema/4309 ; DOI : 10.4000/trema.4309

Ce document a été généré automatiquement le 19 avril 2019.

Trema 


\title{
Montessori et les enfants nomades : forme scolaire et mouvement de l'enfant
}

\author{
Bérengère Kolly
}

«Celui qui compose en marchant est (...) libre d'attaches, sa pensée n'est pas esclave des autres volumes, pas appesantie par les vérifications, pas alourdie par la pensée des autres. Aucun compte à rendre, à personne. Seulement penser, juger, décider. C'est une pensée qui naît d'un mouvement, d'un élan. On y ressent l'élasticité du corps, le mouvement de la danse. Elle retient, elle exprime l'énergie, le bondissement du corps ".

Gros, F. (2009). Marcher, une philosophie, p. 33.

\section{Introduction : libération du mouvement enfantin et développement psychique}

1 La multiplication, depuis les années 2010, de dispositifs issus de la pédagogie Montessori, ou présentés comme tels, semble être en train de modifier les pratiques classiques dans la maternelle française. L'institution scolaire se préoccupe de plus en plus de ces changements, parfois à la marge - ateliers individualisés lors de plages spécifiques parfois plus conséquentes, par la suppression pure et simple des ateliers dirigés qui avaient jusqu'ici construit une des spécificités de la maternelle. Ces modifications peuvent poser un problème de référentiel, pour l'inspection, pour la formation, et pour le référentiel du métier d'enseignant, d'autant que dans certaines académies, les Etudiants Fonctionnaires Stagiaires sont principalement placés en maternelle, et c'est de la maternelle qu'ils construisent leur image du métier.

De tels dispositifs, comme les Ateliers Individualisés de Manipulation, ont un lien parfois très lointain avec la pédagogie Montessori, dans leur contenu comme dans leur approche; ils engagent néanmoins une modification parfois très importante d'une composante de la « forme scolaire » classique, en permettant aux élèves, sur une période déterminée ou sur la journée entière, de travailler de manière individuelle, sur le mode 
du libre choix plus ou moins encadré, et de bouger à leur guise dans l'ensemble de l'espace de la classe. Ils ont ainsi pour premier effet de commencer, peut-être, à réinsérer, dans le regard de l'enseignant, et potentiellement de l'institution, la place du corps de l'enfant dans la classe - un corps "vivant ", " vécu ", et non seulement un corps conçu, pour reprendre les distinctions de Bernard Andrieu (Andrieu, 2016) - et par répercussion, de modifier à la fois le vécu de la classe et celui de l'enseignant. La pédagogie Montessori, proposée dans son orthodoxie ou dans des formes adaptées, engage ainsi une modification ou un questionnement de la forme scolaire traditionnelle, du point de vue du corps de l'enfant, en substituant au corps contraint de l'élève un corps en mouvement.

3 La philosophie occidentale s'est assez peu penchée sur la question du corps, préférant s'attacher à l'âme et ses passions (Descartes), la raison pure (Kant) ou l'entendement (Hume). Le corps semble être aujourd'hui le lieu de deux considérations antagonistes. D'un côté, il est proposé comme "un fardeau dont il faudrait pouvoir se libérer", conception qui, si elle se veut libératrice, continue de réactiver le dualisme philosophique classique du corps et de l'âme, ou encore considère le corps de manière instrumentale ainsi des modules, dans la formation des enseignants, autour des "techniques corporelles » spécifiques du professeur (Jourdan, 2015) qui, tout en réinscrivant le corps dans la pratique de classe, les asservissent aux idées directrices de la conscience de l'enseignant. D'un autre côté, le corps est décrit comme un organisme biologique « déterminant toute conduite ou décision humaine » (Marzano, 2016, p. 3). Dans ce second cas de figure, le corps hégémonique est asservi à un "réductionnisme naturaliste " (Andrieu, 2016) : le prestige réducteur des neurosciences et du cognitivisme en est une des conséquences les plus visibles. Reprenant les travaux de la phénoménologie, en particulier ceux de Merleau-Ponty, Bernard Andrieu propose la construction d'une " écologie corporelle » qui tente de comprendre à la fois le corps vivant - ce que le corps vit de l'intérieur - le corps vécu - ce que je dis de mon corps, qui ne coïncide pas nécessairement avec le premier, comme le notait Wittgenstein - et le milieu dans lequel le corps se trouve. Il propose ainsi l'idée que le corps participe de l'évolution de notre rapport à nous-même, à notre propre corps, et au monde, dans un aller-retour entre sens et cerveau. Il propose ainsi le terme d' » inconscient corporel » :

«L'inconscient corporel est l'ensemble des habitus, techniques du corps, gestes et postures incorporés que nous accomplissons sans nous en rendre compte et qui incarnent notre style singulier. Constitué par notre culture matérielle, l'inconscient corporel (Winnicot, Klein) est fondé sur le concept d'incorporation. Cela pose le problème de la constitution du corps. Il suffirait d'avoir un corps pour incorporer des sensations, des odeurs... Tout ce qui va singulariser la chair (Leib), la constitution biosubjective du sujet » (Andrieu, 2016, p.51)

4 Il est possible, à partir de cette proposition ici seulement esquissée, de réfléchir aux constructions mentales et psychiques induites par le rapport spécifique au corps que propose l'école.

5 A propos du mouvement, Maria Montessori écrit dans la Pédagogie scientifique que ce dernier ne doit pas être considéré comme un divertissement à part, ne modifiant en rien l'organisation de la vie même de l'école et de l'enfant. "Nous avons (...) introduit pleinement l'éducation des mouvements dans l'ensemble unique et inséparable de l'éducation de la personne enfantine" (Montessori, 1909/1952, p. 65). Ce mouvement, parce qu'il est « le sine qua non de la vie » ne peut être ni canalisé ni réprimé ; il devra - et ce sera la tâche de l'éducation - aider l'enfant à « répartir convenablement les énergies et à les laisser se développer convenablement ». 
6 Ce développement est présenté comme indissociablement physiologique et psychique. C'est parce que la doctoresse affirmait que le seul problème pédagogique sérieux devait être celui du développement de la personnalité qu'elle avait fait du mouvement libre une base à la fois de la pédagogie et des dispositifs pédagogiques. Croissance du corps et croissance de l'esprit ou de « l'âme » se répondent l'une l'autre, dans le cadre d'un milieu - nous retrouvons le triptyque de Bernard Andrieu - qui vient non réprimer ou cadrer ce mouvement, mais inciter l'enfant à l'équilibrer. Il faut ainsi comprendre la classe comme un milieu, une « ambiance » qui vient « modifier $^{1}$, c'est-à-dire aider ou détruire » cette double croissance enfantine du « corps qui croît » et de l' » une âme qui se développe. « La double forme physiologique et psychique a une source éternelle: et c'est cela la vie ", écrit-elle ainsi (Montessori, 1909/1952, p. 47). Liberté de mouvement et d'installation, liberté dans l'alternance du repos et de l'activité, liberté dans l'alternance des positions $\mathrm{du}$ corps, mouvement dirigé, contrôlé ou mouvement compris comme levier d'incorporation du savoir dans les activités pédagogiques : chacune de ces déclinaisons participent de «l'extension de la vie enfantine » (p. 48). Car l'enfant possède, écrit Maria Montessori, une capacité à incarner les expériences, les «impressions» qui sont les siennes - c'est la proposition de «l'esprit absorbant » : l'enfant constitue ainsi une « chair mentale » de ce qui l'environne ${ }^{2}$.

7 Ces différentes considérations, trop rapidement présentées, doivent permettre de comprendre que le matériel montessorien ne se contente pas d'exercer à la motricité fine, de réguler le mouvement de l'enfant ou de faire apprendre en bougeant; ce serait là réduire considérablement la portée et le sens de la pédagogie en la restreignant à un aspect utilitaire, ou en l'enfermant par un mécanisme de réduction naturaliste.

8 La libération du mouvement de l'enfant dans la classe d'une part, l'exercice au mouvement contrôlé dans le matériel d'autre part relèvent tous deux clairement d'un objectif d'expansion de la personnalité, c'est-à-dire des forces conscientes et inconscientes de l'enfant. Le mouvement du corps répond et incarne l'élan de vie, qui est tout à la fois un désir de vie, de croissance et de savoir. Cette notion d'élan, que l'on trouve en particulier sous la plume de Bergson, permet de comprendre l'intrication entre conscience et mouvement.

« La conscience, originellement immanente à tout ce qui vit, s'endort là où il n'y a plus de mouvement spontané, et s'exalte quand la vie appuie vers l'activité libre ", écrit Bergson ; le mouvement est ce qui permet et ce qui caractérise la conscience ; et la conscience un mouvement, une force, qui «s'insèr[e] dans la nécessité et la tourn[e] à son profit » (Bergson, 1911/2009, p. 11).

Autrement dit, le mouvement libre a pour objectif, qui est aussi celui de la pédagogie dans son entier, de développer la puissance de l'enfant, physique, psychique, intellectuelle, spirituelle, puissance qui est aussi régulation des aptitudes.

10 L'ensemble de ces propositions se trouve dans les textes montessoriens; quant à leur mise en pratique, elles ne trouvent leur forme radicale - dans le double sens d'écho à ce qui est constitutif de la pédagogie, et sans exception ou atténuation dans la pratique - que dans les pratiques que l'on peut qualifier, à la suite d'Henri Louis Go à propos de l'École Freinet de Vence, de «correctes» (Go, 2007). Nous proposons donc, au regard des évolutions récentes par lesquelles nous avons ouvert cet article, d'explorer les modifications de pratiques que de telles considérations engagent.

11 Le terrain sur lequel nous nous basons est celui d'une maternelle Montessori située à Lyon, dans le cadre d'un établissement catholique sous contrat avec l'État qui propose 
cette pédagogie pour l'ensemble de ses classes en primaire. Ces classes sont composées en moyenne d'une trentaine d'élèves, encadrées par un ou deux enseignants. L'enseignante avec laquelle nous travaillons a plus de vingt-cinq années d'expérience dans la pédagogie Montessori. Elle est titulaire du diplôme 3-6 ans validé par l'Association Montessori Internationale, et engagée depuis plus de trois ans dans un processus de formation d'autres enseignants - processus dont bénéficie la deuxième enseignante de cette classe. Le travail de recherche que nous avons engagé avec elle est continu depuis le début de l'année scolaire 2014-2015. Il consiste dans un travail d'immersion régulier, d'observations participantes de type ethnographiques, d'entretiens et de prises vidéo. Sur le modèle des «cliniques" proposées par Gérard Sensevy, cette recherche fonctionne dans l'appui sur l'expertise de l'enseignante, et sur un échange régulier entre recherche universitaire et pratique de classe.

12 A partir de ces observations, nous proposons d'esquisser les modifications de la forme scolaire qui peuvent être induites par cette présence du corps de l'enfant en mouvement dans l'organisation du collectif. Nous proposons en particulier de rendre compte du régime spatio-temporel spécifique qui est celui des enfants de maternelle Montessori, ce dernier entrant en contradiction ou en friction avec la forme scolaire traditionnelle.

Pour autant, il nous faudra tenter de définir ce que peut être une pratique Montessori, ou à défaut, de circonscrire les questions et les problèmes qu'une telle tentative de définition soulève - ce qui constituera la première partie de notre propos.

Nous nous pencherons ensuite, dans une deuxième partie, sur le régime spatio temporel proposé dans une classe Montessori, qui constitue l'enfant comme une trajectivité au sens donné par Berque : celui d'une inscription dans le monde, une relation entre objectivité et subjectivité, la manière dont nous habitons l'espace, «le domaine sur lequel nous agissons, et qui porte les marques de cette action, mais (...) aussi le domaine qui nous affecte, et auquel nous appartenons de quelque manière » (Berque, 2009, p. 142).

15 Nous en étudierons, dans une troisième partie, l'effet premier que produit ce régime spatio-temporel : les enfants marcheurs, ou les enfants nomades.

\section{Comment circonscrire une pratique Montessori ? Problèmes et enjeux}

\section{Qu'est-ce qu'une « pratique Montessori »?}

Dire qu'une classe «fonctionne en Montessori », ou encore qu'une classe, dans le public, le privé, en contrat ou non, « est Montessori » est une affirmation rarement interrogée, comme si le déclaratif suffisait à définir la, ou plutôt les pratiques. Car des pratiques correctes aux adaptations plus ou moins lointaines de la pédagogie, des "Ateliers Individualisés de Manipulation » mis en place en maternelle ou des pratiques Alvarez qui se défend elle-même de pratiquer une pédagogie Montessori ${ }^{3}$ tout en en recyclant de fait le matériel et l'essentiel de ses habitudes, l'hétérogénéité, de pratiques et d'esprit, sont de fait de mise. Reconnaître ces hétérogénéités semble nécessaire pour reconnaître, d'une part, que les «pratiques Montessori » recouvrent des expériences pédagogiques diverses, que ce soit dans les techniques employées, le respect du matériel originel, la formation suivie ou encore l'esprit dans lequel la pédagogie est pratiquée ; pour affirmer, d'autre part qu'il existe dans le même temps des pratiques "correctes" qui 
correspondent à la fois à l'esprit et à la lettre de la pédagogie - sans présumer une quelconque supériorité des unes sur les autres.

17 Jusqu'à une date très récente - nous y revenons un peu plus loin - le mouvement Montessori en France et les formations qu'il proposait ${ }^{4}$ restaient attachés, d'abord, en termes techniques, au contenu habilité par l'Association Montessori Internationale; ensuite, en termes d'exigences philosophiques, à une vision de l'enfant, de l'adulte et de leur relation; enfin, en termes de cadrage, à un certain nombre d'exigences, comme une formation d'une durée conséquente, en présentiel, prodiguée par une personne habilitée et d'expérience; un esprit de corps et une posture spécifique du maitre - nous y reviendrons -; une méfiance, voire un refus des mixages et des adaptations de la pédagogie. Ces principes peuvent donner lieu à un premier socle pour penser les pratiques correctes - socle par ailleurs remis en question par un certain nombre d'initiatives actuelles, qui modifient sans doute le rapport que le mouvement entretient avec son orthodoxie.

\section{Montessorisme, mouvement Montessori et diffraction de la pédagogie}

Car ces questions ne sont pas nouvelles dans l'histoire du mouvement. La pédagogue et ses proches se sont rapidement posé des questions similaires face au phénomène de « diffraction » (Kolly, 2018) du montessorisme des années 1910. Ce terme, emprunté à la physique des ondes, permet de décrire un phénomène spécifique de diffusion de la pédagogie, complémentaire de ceux de la dissémination, de la circulation ou de la réfraction (Droux, Hofstetter, 2015). La diffusion de la pédagogie Montessori à grande échelle, de manière précoce, a très vite incité des instituteurs ou des éducateurs à monter des classes «Montessori », par de simples lectures, sans nécessairement avoir été formés officiellement, ou de manière très rapide - les premiers cours semblent d'ailleurs avoir été assez succincts. Ces personnes ont été amenées à assurer elles-mêmes des cours et des conférences; et ces personnes ont à leur tour fait des émules, qui ont également formé, etc. Ce système de diffraction est extrêmement efficace d'un point de vue communicationnel, ou médiatique, et à ce titre, les années 2010 en France semblent réactiver, toutes proportions gardées, un phénomène similaire ayant eu lieu dans les années 1910. Mais de fait, cette diffraction pose des problèmes de fond et de forme : celui d'assurer un minimum de perte dans les pratiques et les intentions pédagogiques question qui est au cœur par exemple, de la diffusion actuelle de la pédagogie Montessori par le biais d'Alvarez en France; celui de se protéger des pratiques incorrectes - question qui peut être soulevée par les adaptations lointaines de la pédagogie dans la maternelle classique comme les Ateliers Individualisés de Manipulation.

Tout ceci signifie que, dès ses débuts, la pédagogie Montessori semble s'être scindée en deux collectifs. Le premier collectif, à qui nous proposons de réserver le terme de "mouvement Montessori», est un collectif nommé par Decroly en 1924 le camp " orthodoxe ». Du vivant de la pédagogue, c'est un petit réseau, constitué de personnalités proches, tenant des écoles au fait de la pédagogie appliquée stricto sensu, et de ses évolutions. Après la disparition de la pédagogue, l'A.M.I. prend le relais, pour conserver un réseau restreint et fermé - celui des " puristes ", si l'on veut. Le second collectif, à qui nous proposons de donner le terme de "montessorisme ${ }^{5}$ ", est un mouvement de pratiques et de pensées à partir de la pensée montessorienne, mais en dehors des réseaux 
autorisés stricto sensu, de la pédagogue ou des institutions qui s'y rapportent. Surtout, ce collectif entretient un rapport plus ou moins relâché à la lettre de la pédagogie ; il permet et encourage les mixages avec d'autres pédagogies ou démarches, engage des libertés dans les pratiques et dans l'esprit. Dans l'entre-deux guerres, le montessorisme - qui concerne des enseignants du classique, ou de l'Education Nouvelle, comme Madeleine Guéritte $^{6}$ - a davantage la faveur des éducateurs de l'Éducation Nouvelle; le mouvement Montessori, pour sa part, reste en distance de ces réseaux, en dépit de la large diffusion de la pédagogie.

\section{Les années 2000 : une rupture dans l'histoire du mouvement Montessori?}

Pour autant, la comparaison entre l'engouement des années 1910 et celui des années 2010 reste à relativiser. La principale raison tient sans doute au fait que l'appétence nouvelle des enseignants du classique pour la pédagogie vient perturber ce fonctionnement en deux collectifs distincts - sachant que le premier, le "mouvement Montessori », restait jusqu'ici relativement confidentiel, et d'une socialisation spécifique, et que le second, le " montessorisme ", avait quasiment disparu des écoles traditionnelles.

21 L'arrivée de Céline Alvarez dans les années 2010 vient modifier cette distribution. La particularité de cette enseignante temporaire (elle enseigne trois années à Gennevilliers) est d'avoir été formée selon l'orthodoxie, mais de n'en revendiquer ni l'appartenance ni l'héritage. La rupture qu'elle effectue n'est donc pas tant pédagogique que morale. Pédagogiquement, elle présente une expérience montessorienne classique ; moralement, en revanche, elle refuse toute appartenance à un groupement, et toute dette envers un héritage ou une tradition qui la précèderait, en proposant une démarche centrée sur son expérience. Elle pourrait ainsi, potentiellement, proposer une voie nouvelle, qui reviendrait à faire disparaître le nom «Montessori » de la pédagogie, ce qui constitue sans doute une nouveauté dans l'histoire française du mouvement.

Pour l'instant, cette disparition ne semble pas encore à l'ordre du jour. Car la déstabilisation de la partition en deux collectifs, pour l'instant, semble aller dans le sens d'une diffusion de la pédagogie Montessori. En proposant une démarche supposée aisément applicable par tous - et en minimisant sans doute les difficultés pédagogiques qu'elle engage - Alvarez répond au besoin de rénovation de l'école, et aux désirs pratiques des enseignants. Elle amène ainsi les praticiens classiques à se tourner vers le montessorisme. Mais dans le même temps, un certain nombre de ces praticiens éprouvent la nécessité d'une formation de qualité, et dans une forme plus classique, dépassant la seule information horizontale, pour répondre aux questions pratiques qu'ils se posent alimentant cette fois non plus le montessorisme, mais une orthodoxie des pratiques, qui, de fait, change de visage. C'est ainsi que l'association «Public Montessori », née en 2015, a été fondée par des enseignants formés à l'Institut Supérieur Maria Montessori (I.S.M.M.) : elle entend proposer des contenus orthodoxes à des praticiens du public ${ }^{7}$.

Parions donc que la question de l'orthodoxie ne se posera plus, à court terme, sur la question de la formation des enseignants, mais sur la formation de formateurs. Les critères jusqu'ici adoptés de cette formation - cooptation stricte et le respect d'une forme de confidentialité autour des contenus de formation - sont remis en cause par la démocratisation du mouvement. C'est donc au sein de cette formation que la diffraction 
risque de se réactiver, posant la question des critères pour former à la pédagogie (type de formation, origine de la formation, expérience de classe du formateur par exemple).

Jusqu'ici, le mouvement Montessori n'a pas engagé d'habilitation, de contrôle ou de labellisation des classes elles-mêmes, ou des praticiens à partir de leurs pratiques - il n'existe pas, en d'autres termes, d'inspection montessorienne. Il existe de fait une recension des écoles ou des classes Montessori, basée sur l'adhésion à l'Association Montessori de France; mais cette recension peut surtout donner une idée du réseau montessorien en France. Si le choix est fait d'un respect des pratiques correctes, une telle cohérence de pratiques, dépassant la seule formation des enseignants pour interroger le suivi des pratiques et la formation de formateurs sera sans doute nécessaire. Dans le cas contraire, la diffraction idéologique et philosophique serait à craindre non en dehors, mais à l'intérieur du mouvement orthodoxe lui-même.

\section{La classe Montessori : un régime spatio temporel alternatif}

\section{Une organisation non disciplinaire}

Dans le numéro 12 de la revue Pour l'ère nouvelle, en 1924, Ovide Decroly établit un historique et un bilan des expériences montessoriennes menées à La Haye dans les années 1920. Décrit «le règne du chaos" pour des enfants «mis en face du matériel et abandonnés à eux-mêmes " (Decroly, 1924, p. 62). Il dénonce de cette manière l'absence d'intérêt que les enfants manifesteraient spontanément pour le matériel appliqué dans la stricte observance de l'orthodoxie montessorienne, et la trop grande possibilité qu'ont les enfants de ne "rien faire » - engendrant précisément le «chaos ». L'organisation d'une classe Montessori, ainsi que les principes sur lesquels elle se fonde semblent en effet relever, sinon du « chaos », du moins d'un régime explicitement non-disciplinaire.

L'école comme "institution disciplinaire", telle que nous la trouvons décrite chez Foucault possède une caractéristique essentielle: la segmentation, le «quadrillage » de l'espace et du temps, dans un objectif de discipline et de dressage des corps. L'espace est fragmenté, ce qui permet, d'une part, d'encadrer la circulation et les mouvements de manière à ce qu'ils soient toujours prévisibles. Ceci permet, d'autre part, « d'établir des cloisons étanches entre les individus » et « des percées de surveillance continue » (p. 203). Le maître, en particulier, règne sur ce quadrillage, en distribue les places, en permettant ou refusant le mouvement ${ }^{8}$. Le système de "rang", enfin, vient intriquer valeurs et normes, rendant explicite la hiérarchie, mais aussi les écarts, le " non-conforme », « tout ce qui est inadéquat à la règle ", le rang devenant alors le témoin de la grâce ou de la disgrâce. La fragmentation du temps, pour sa part, augmente «la maîtrise d'une durée qui échappe "; il s'agit d'en augmenter son utilité, et de pourchasser l'oisiveté, qui est pourchassée. «L'emploi du temps devait conjurer le péril de le [le temps] gaspiller - faute morale et malhonnêteté économique ", écrit Foucault (Foucault, 1975, p. 178). Dans cette institution disciplinaire, pas de longue durée, mais la scansion régulière et la division de l'emploi du temps; à l'espace vide où s'effectuent mouvements et regroupements s'opposent la division et la subdivision entre cellules dont la circulation est réglée par le regard hiérarchique. Cette organisation spécifique de l'école constitue une " configuration historique particulière ", celle de la «forme scolaire », en continuité avec 
l'école classique telle que nous la connaissons, comme le propose Guy Vincent (Vincent, $1980 ; 1994)$.

\section{L'espace et le temps montessorien, trajectoires enfantines} Les éducateurs pointent, midi et soir, ou au fur et à mesure quand ils le peuvent, les présentations effectuées, les activités répétées, les activités acquises ou en cours d'acquisition. Mais au matin même, ni emploi du temps fixe, ni plan pour le travail : les présentations de matériel se tissent au hasard ou à l'opportunité des croisements des marcheurs et des trajectoires, que ce soient celles des adultes ou celles des élèves. Il ne faudrait pas y voir une renonciation éducative, mais plutôt une exigence éthique, qui laisse à l'enfant la direction - donc aussi la responsabilité, et pourquoi pas la charge - de son emploi du temps. Chaque enfant et chaque adulte sont des trajectoires en mouvement 
perpétuel, imprévisible extérieurement, qui projette une réflexion ou un travail personnel ou collectif. La rencontre, en particulier entre adultes et enfants, peut aussi être à l'initiative de l'enfant, qui vient régulièrement solliciter l'éducateur, pour une présentation, pour une aide, pour une précision; elle devient dans tous les cas celle d'un croisement des disponibilités, au sens philosophique et éthique, et pourquoi pas psychologique du terme. Pour cette raison, les regroupements imposés sont rares, et fonctionnent toujours, à moins d'une raison exceptionnelle, sur le mode de l'adhésion libre.

31 Ainsi, la double continuité spatiale et temporelle des classes montessoriennes répond au quadrillage disciplinaire de la forme scolaire classique : les enfants sont des trajectoires en mouvement perpétuel; le temps y est moins capitalisé que déployé. Le temps de l'enfant montessorien n'est par ailleurs pas envisagé de manière continue, mais plutôt par cycles et par périodes de sauts successifs, des alternances entre maturations silencieuses et "explosions psychiques», celles de l'écriture notamment (Montessori, 1909/1970, p. 200) - une sorte de «tension dans la durée » très bergsonienne, qui décrit un phénomène d'accumulation d'énergie se projetant ensuite dans l'action - tout comme la flèche, qui est d'autant plus puissante que le fil était tendu vers l'arrière (Bergson, 2009, p. 17).

32 La mention de Bergson n'est pas fortuite; car si pour le philosophe «la conscience, originellement immanente à tout ce qui vit, s'endort là où il n'y a plus de mouvement spontané, et s'exalte quand la vie appuie vers l'activité libre», le mouvement, l'activité libre est ce qui permet et ce qui caractérise la conscience; et la conscience peut, en retour, être définie comme un mouvement, une force, qui « s'insèr[e] dans la nécessité et la tourn[e] à son profit » (Bergson, 1911/2009, p. 11). Le mouvement perpétuel de l'enfant dans la classe répond ainsi à une préoccupation d'ordre philosophique autant que psychique : la marche de l'enfant vient accompagner non seulement la pensée, le projet, mais aussi l'émergence de la conscience, de « l'élan » de l'enfant vers le travail.

\section{Les enfants marcheurs}

En observation dans la classe maternelle que nous avons présentée plus haut, nous avons fait le choix de cartographier les trajectoires de plusieurs élèves sur la durée d'une plage continue de travail, matin ou après-midi ${ }^{9}$. Ces trajectoires ont été, soit tracées en direct, soit reconstituées à partir de photographies prises à raison de 2 à 3 photographies par minute. Nous avons ensuite, à partir des notes prises en parallèle, reconstitué non seulement les trajectoires enfantines et leurs objectifs, mais également leur correspondance avec les périodes de travail, concentration, de déconcentration, d'échanges avec la vie de la classe ou encore de ce que nous avons nommé les «errances».

\section{Plusieurs régimes d'existence spatio-temporelle}

Ainsi de Javier, 4 ans et demi, que l'on a dessiné en train de marcher dans la classe, pendant les deux heures du matin : 


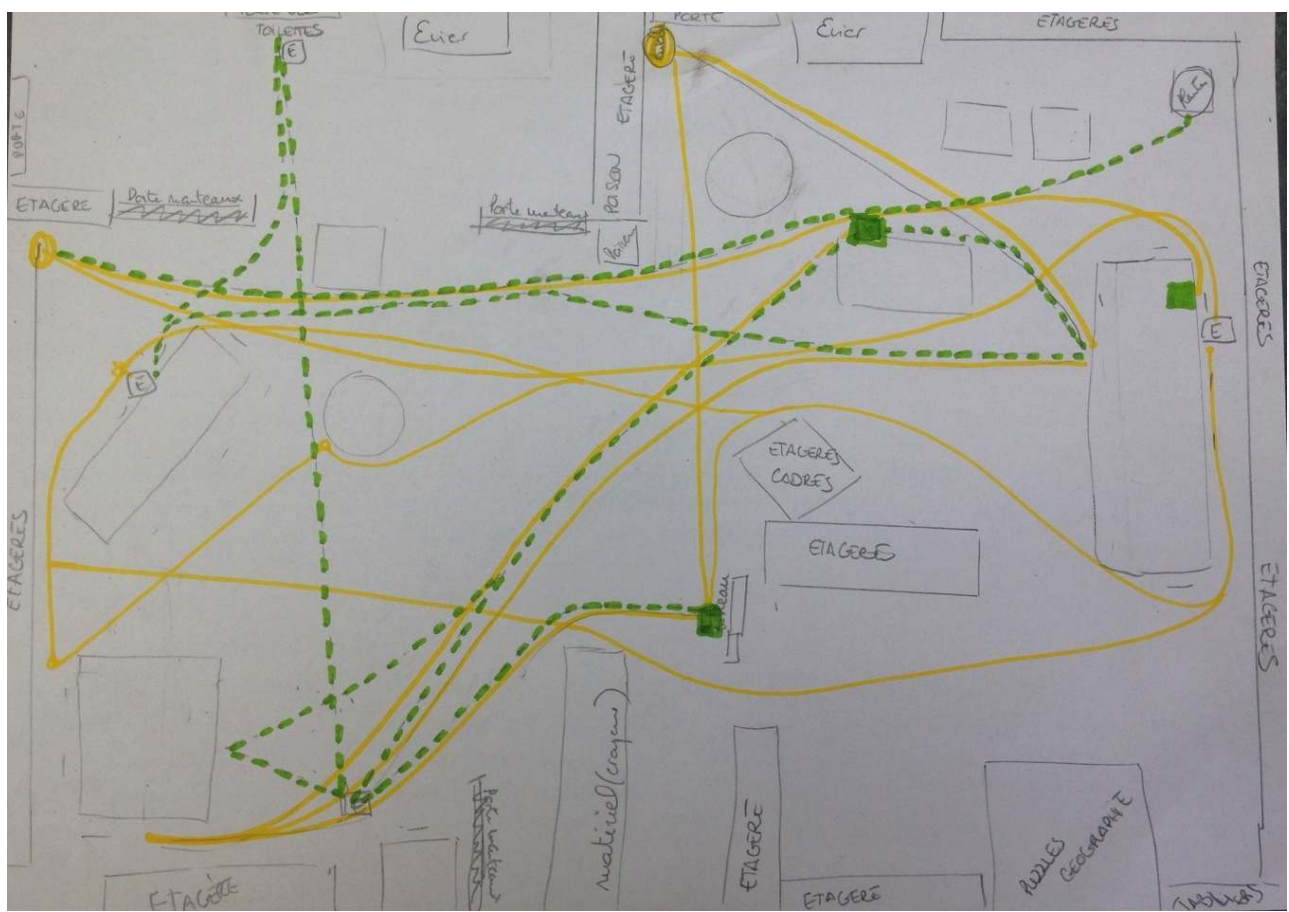

Une telle cartographie doit tout aux cartes composées par Fernand Deligny, qui rend compte, dans son centre des Cévennes, des "régimes d'existence» des enfants autistes qu'il accueille, et qui ne parlent pas. Une telle cartographie, chez Deligny à la frontière entre le saisissement poétique et l'œuvre d'art, ne vient rien démontrer, sinon l'existence, au moins, de « deux régimes d'existence spatio-temporelle » (Fernand Deligny / Alvarez de Toledo, 2013, p. 1). Les trajets possédant un objectif utilitaire identifiable - commande d'un adulte, ou, pour le cas de Javier, sollicitation d'une aide, en pointillés sur la carte ; et les trajets sans objectif utilitaire identifiables, qui peuvent apparaître comme sans raison, les errances, les « lignes d'erre ", écrit Fernand Deligny, identifiées sur notre carte par les traits pleins. Ces errances sont typiques des enfants «marcheurs» montessoriens : elles conditionnent le travail libre, et en sont sans doute une trace; elles permettent la création en encourageant, dans le corps même, «l'élan » qui caractérise pour Montessori tout enfant - élan vers la croissance et vers le travail.

Les enfants marcheurs sont des enfants qui expérimentent ainsi plusieurs types de rapport à l'espace, au temps, donc aussi à autrui - aux adultes, à leurs pairs - et à euxmêmes. Le même Javier expérimente, au cours de cette matinée de janvier 2016, au moins trois rapports différents au temps. Voici l'organisation de cette matinée.

Tableau 1 : Organisation de la matinée de Javier

\begin{tabular}{|l|l|}
\hline Matinée de Javier & \\
\hline Travail 1 (écriture / dessin) & 52 minutes (alternance : travail individuel ; travail à deux) \\
\hline Errance 1 & 3 minutes \\
\hline Travail 2 (écriture au tableau) & 14 minutes (dont un épisode de conflit de 2 minutes) \\
\hline
\end{tabular}




\begin{tabular}{|l|l|}
\hline Errance 2 & 30 minutes (dont deux épisodes, et 15 minutes "d'attente" \\
\hline Travail 3 (présentation / travail) & 15 minutes \\
\hline
\end{tabular}

On peut y repérer trois types de moments liés à l'enfant lui-même.

En premier lieu, Javier expérimente ce que nous pourrions nommer les «focales », c'està-dire des moments où la concentration de l'enfant se focalise sur le travail, seul, à plusieurs, ou en alternance - comme c'est le cas ici. Javier démontre d'ailleurs une grande capacité de concentration, puisqu'il reste à son travail (écriture de mots et dessins correspondants aux mots) pendant quasi une heure - une heure où il reste néanmoins en mouvement: il change de place trois fois, fait des courtes pauses, a des moments de déconcentration, ou des moments où son regard se fait plus vague. Cette première expérience vécue est celle du travail, du rapport à soi, à la concentration, au but que l'on se fixe et que l'on accomplit, ou non.

38 En deuxième lieu, Javier semble expérimenter ce que nous pourrions appeler les « épisodes », c'est-à-dire des moments de vie de la classe, du collectif des enfants. Ce matin-là, Javier participe à trois épisodes: la récupération d'un objet perdu dans la poubelle, que plusieurs enfants s'évertuent à récupérer; la recherche d'un petit papier perdu dans les affaires d'un camarade; un conflit avec d'autres enfants à propos du chevalet qu'ils partageaient alors. Ces « épisodes» non interrompus par l'enseignante, sont des souffles dans la succession des actions de l'enfant; ils participent également de la constitution d'un collectif vivant.

En troisième lieu, Javier démontre qu'il vit des moments spécifiques, que nous voudrions décrire plus précisément ici, et que nous nommons, à la suite de Deligny, des « errances ». L'errance possède le double sens de déambuler sans but, mais aussi de se tromper (Berthet, 2007, p. 10). L'errance est ainsi une expérience qui est aussi « danger », « perte de soi-même "; elle peut être vue comme un comportement déviant - quel est cet enfant qui ne travaille pas? L'observation de Javier - et d'autres enfants, dont nous ne ferons pas la description faute de place - montre que si l'errance est une perte - perte de concentration, perte de repères et d'objectifs - elle est aussi un éloignement qui permet une nouvelle quête, une découverte d'autre chose. L'enfant marcheur, en errant, est aussi en recherche: dans ce sens, dans l'errance, «l'objectif n'est pas de se perdre mais au contraire de se trouver » (op. cit.).

\section{Les errances}

Lors de la première errance, Javier se dirige vers le fond de la classe : il s'arrête quelques poignées de secondes près d'un groupe en travail près de l'enseignante ; il paraît un peu ailleurs, peu concentré. Puis il repart, et se dirige cette fois vers le chevalet, où se trouve un groupe de quatre enfants. Ces enfants sont en train d'écrire, collectivement, tous les noms des enfants de la classe sur la face blanche du tableau. Javier s'approche, les regarde, et se met lui-même à écrire sur la face noire du chevalet. L'errance semble lui avoir donné, dans ce cas, une idée de travail ; mais ce travail n'est pas choisi au hasard, puisqu'il correspond et répond au premier travail de la matinée (l'écriture). L'enseignante pourra préciser, par la suite, que Javier était dans une période où l'écriture était très importante pour lui, et lui prenait une bonne partie de son temps. 
41 La deuxième errance commence de nouveau par l'observation d'autres enfants; elle est interrompue par la proposition, par l'enseignante, d'une présentation de mathématiques. Javier va donc passer les 30 minutes d'errance à préparer le matériel pour la présentation; mais cette préparation est entrecoupée par des observations, des trajets qui paraissent non utilitaires, et par les deux épisodes de la poubelle et du petit papier. Une fois installé à sa table, Javier attend l'enseignante, entre-temps prise par d'autres enfants, en interagissant avec deux autres enfants assis à la même table. Enfin, il bénéficie d'un temps de présentation individualisée de 7 minutes, suivi d'un temps de travail individuel - mais en interactions avec le même camarade avec lequel il avait travaillé en tout début de matinée, jusqu'à la fin du temps de travail.

42 L'enjeu de telles errances est plus important qu'il n'y parait. Outre l'insertion, dans un cadre institutionnel souvent rigide, de moments souples valorisant une attention flottante, une concentration moindre, et des moments non explicitement utiles (les enfants ne « font rien », extérieurement) ces errances permettent plusieurs choses.

43 Elles permettent, d'abord, les observations réciproques entre enfants, nécessaires à une émulation, par ailleurs favorisée par le mélange des âges, donc aussi des niveaux, des activités et des centres d'intérêt. Les errances, en favorisant l'observation de l'autre, permettent à l'enfant de se projeter, dans le temps, mais aussi dans ses capacités ou ses désirs. Ceci n'est possible qu'en rendant à l'enfant sa capacité de mouvement, donc aussi de marche : l'enfant marcheur, l'enfant nomade, pour reprendre un terme deleuzien, est celui qui met à l'épreuve sa volonté, qui la confronte à celle des autres, mais aussi qui met à l'épreuve son désir.

44 L'enfant marcheur peut alors être comparé à un insecte butinant les fleurs qui se présentent à lui, participant ainsi à l'œuvre naturelle de reproduction des plantes. Il s'agit bien de désir, au sens deleuzien d'une force vitale, d'une énergie productrice et créatrice ; mais il s'agit également de l'édification de la personnalité : le mouvement, écrit Maria Montessori, entraîne un "travail constant qui édifie $1[\mathrm{a}]$ personnalité » en la provoquant « entièrement par les décisions » (Montessori, 1970, p. 151).

45 Les errances permettent ensuite au corps de l'enfant d'expérimenter et de se confronter à son milieu. Pour Bernard Andrieu, « la pratique corporelle influence la compréhension de soi par le corps » (Andrieu, 2008, p. 257) : se mouvoir entraîne une modification tout à la fois physique, mentale, affective et relationnelle - à l'image des arts martiaux, qui travaillent non seulement le corps physique, mais également, dans l'exercice, la pratique de valeurs, de dispositions mentales, voire même une philosophie de vie. En ce sens, ne pas dissocier le savoir du mouvement engendrerait un aller-retour entre l'action de l'enfant sur son environnement, et l'action de l'environnement, par l'action de l'enfant, sur lui-même; en d'autres termes, un enfant marcheur est un enfant qui prend conscience à la fois du monde et de lui-même agissant sur ce monde. Marcher dans la classe deviendrait une condition nouvelle, corporelle, de la réflexivité et d'une interrogation sur le sens du savoir.

Enfin, l'errance pose la question de la subversion. Deleuze, philosophe de la "pensée nomade ", écrit que l'organisation - dans l'État, dans la classe - est le levier essentiel du pouvoir, car l'organisation est une machine à "écraser le désir ». A l'inverse, le nomadisme, l'essaimage, parce qu'ils s'opposent aussi à l'organisation, permettent une libération du désir, d'une énergie productrice et créatrice. Cette dernière piste, qui mériterait bien entendu d'être approfondie, d'autant qu'elle s'accorde avec un cadrage 
relatif d'une forme de l'activité enfantine, verrait les enfants marcheurs comme une proposition philosophique, et pourquoi pas politique, d'une libération du désir, ou dans des termes plus montessoriens, de la «puissance» de l'enfant. Car la pédagogie montessorienne se présente explicitement comme devant développer chez l'enfant une puissance multiple - physique, psychique, intellectuelle, spirituelle; elle se décline sous l'image de la santé, psychique autant que physique, la méthode visant à un enfant « au caractère robuste, à la discipline profonde", écrit-elle, ayant acquis une "santé intérieure (...) résultat de la libération de l'âme » (Montessori, 1909/1952, p. 120). Il en va alors du projet politique montessorien: celui d'une «révolution de l'individu», levier nécessaire, écrit Montessori, pour pouvoir construire la seule révolution que la pédagogue appelle de ses vœux : la révolution de la non-violence.

\section{Conclusion}

47 Cet article entendait revenir sur une particularité des classes montessoriennes, l'enfant marcheur, ou l'enfant nomade, et sur un de ses éléments cruciaux, l'errance. Ces phénomènes se voient à répétition, bien entendu de manière différenciée selon l'âge et l'avancée des enfants, dans les classes à la pédagogie dite orthodoxe; mais ces phénomènes s'observent aussi dans des pratiques non orthodoxes, voire lointaines, comme les Ateliers Individualisés de Manipulation. Si le contenu de ces ateliers est très éloigné du matériel montessorien, et si la posture des enseignants est souvent assez classique, donc là aussi très différente de la posture montessorienne, leur organisation de l'espace et du temps reste parfois comparable. En cela l'étude des pratiques strictes possède un intérêt: celui de considérer les pratiques génériques qui pourraient être pensées, de ces pratiques alternatives, vers l'école pour tous.

Les élèves, pour trente minutes, une heure, ou pour une partie plus importante de la journée, jouissent en effet d'une liberté dans leurs mouvements, dans leur installation, et parfois dans leur temps. Or on observe - nous avons observé, il est vrai sur un échantillon assez faible - des phénomènes similaires d'errance. Adel, observé dans une maternelle REP + en Lorraine ${ }^{10}$, témoigne, comme Javier, de deux moments de travail, entrecoupés d'un temps d'errance. Pendant ce temps d'errance, Ibrahim n'observe néanmoins pas les autres enfants, mais exerce son choix, passant d'un endroit à un autre, semblant hésiter entre telle ou telle activité, jusqu'à effectuer son choix. Son errance, recueillie de la même manière que celle de Javier, et cartographiée ci-dessous, ressemble davantage à un temps de latence entre deux activités - sachant que le temps total de travail est d'une heure, et non de deux heures. 


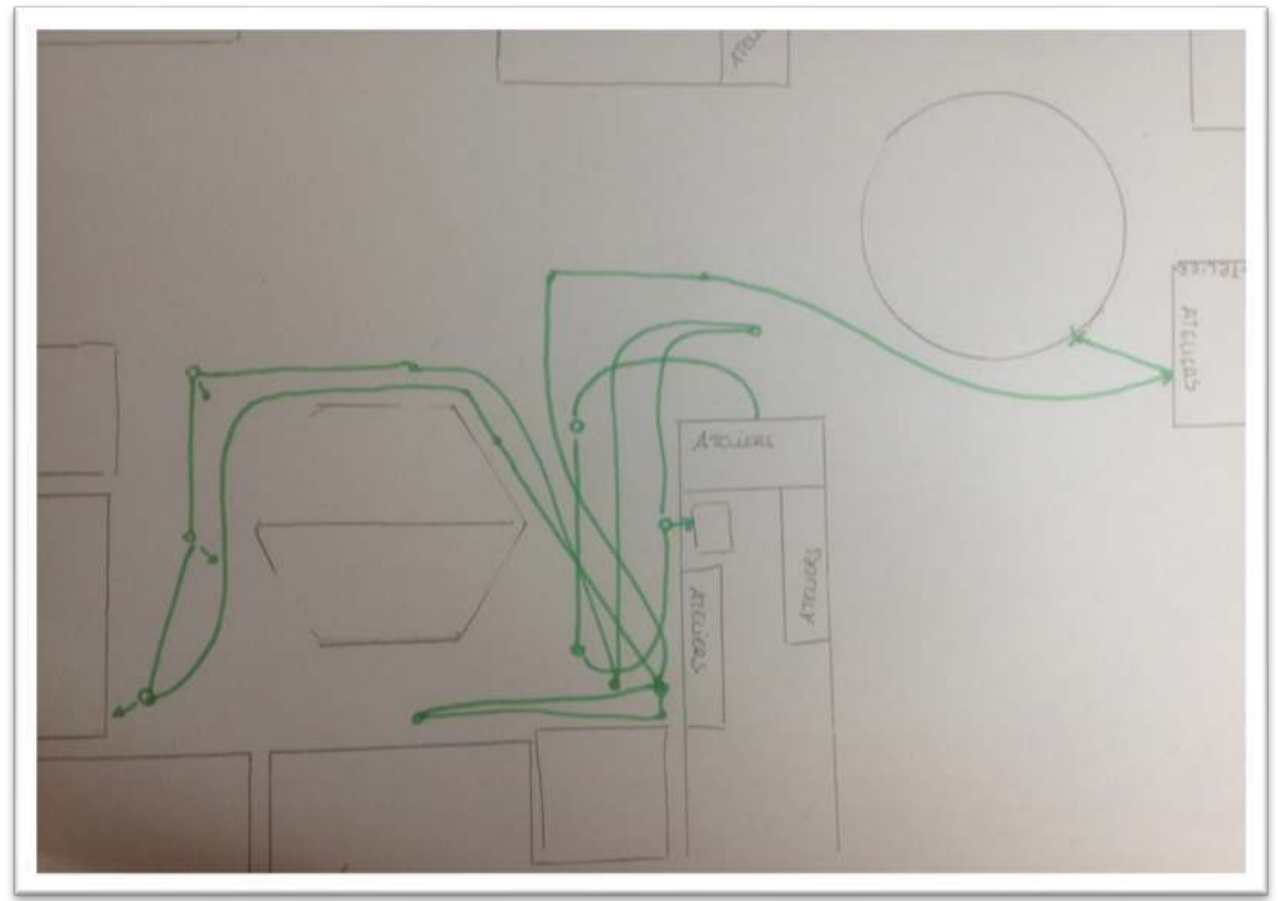

Il est bien entendu impossible de tirer des conclusions générales de ces deux exemples.

Au regard de la question qui était la nôtre au début de cet article, nous pouvons peut-être énoncer deux éléments.

Le premier est que dans un cadre traditionnel ou classique, l'espace et le temps restent généralement fragmentés - pour des raisons sans doute parfois encore disciplinaires, mais aussi en remède d'une attention faiblissante de l'élève, de sa fatigue ou de son inattention. Le cadre montessorien, en abolissant la fragmentation de l'espace-temps de l'enfant, entend retourner l'argument : le travail, qui était celui du maittre, devient celui de l'enfant. En cela, la proposition montessorienne, qu'elle soit orthodoxe ou plus libre, vient bien heurter ou questionner la forme scolaire traditionnelle. Peut-être s'agit-il bien ici d'une proposition démocratique, ou plus démocratique, tendant à une égalité entre adulte et enfant: elle engage en effet le maître à se préoccuper de l'activité propre et spontanée de l'enfant, et donc à effectuer un «déplacement radical» de son activité (Montessori, 1909/1952, p. 119).

52 Le second élément est, si nous considérons que l'exemple présenté ici peut être pris comme un "exemple exemplaire », pour reprendre la démarche de Gérard Sensevy ${ }^{11}$, qu'il faut comprendre, cette fois d'un point de vue épistémologique, que l'étude de pratiques radicales, orthodoxes, ou « correctes » est sans doute un levier pour étudier les pratiques lointaines et les adaptations d'une pédagogie : non pour les hiérarchiser, mais au contraire pour en mieux saisir la teneur et les enjeux. 


\section{BIBLIOGRAPHIE}

Montessori, M. (1909/1952). Pédagogie scientifique, tome 1. Paris : Desclée de Brouwer.

Montessori, M. (1909/1970). Pédagogie scientifique, tome 1. Paris : E.S.F.

Decroly, O. (1924). Expériences d'éducation nouvelle à La Haye. Pour l'ère nouvelle. n¹2, pp. 62-65.

Alvarez de Toledo, S. (2013). Cartes et lignes d'erre. Traces du réseau de Ferdinand Deligny, 1969-1979. Paris : L'arachnéen.

Andrieu, B. (2016). Sentir son corps vivant. Paris : Vrin.

Andrieu, B. \& Boëtsch, G. (Dir.). (2008). Dictionnaire du corps. Paris : CNRS Éditions.

Berthet, D. (2007). Figures de l'errance. Paris : L'Harmattan.

Bergson, H. (2009). L'énergie spirituelle. Presses Universitaires de France.

Berque, A. (2009). Écoumène. Introduction à l'étude des milieux humains. Paris : Belin.

Caron, J.C. (1999). A l'école de la violence, châtiments et sévices scolaires dans l'institution scolaire au XIXe siècle. Paris : Aubier.

Deleuze, G. (2002). Ile déserte et autres textes - 1953 - 1974. Paris : Minuit.

Droux, J. Hofstetter, R. (dir). (2015). Globalisation des mondes de l'éducation. Circulations, connexions, réfractions. XIXe-XXe siècles. Presses Universitaires de Rennes, Collection « Histoire ».

Foucault, M. (1975) Surveiller et punir. Paris : Gallimard.

Go, H.L. (2007). Freinet à Vence. Vers une reconstruction de la forme scolaire. Presses universitaires de Rennes.

Gros, F. (2009). Marcher, une philosophie. Carnets Nord.

Gutierrez, L. Savoye, A. (2018, à paraître). Sauver les jeunes générations du désastre éducatif et sanitaire : les combats de Madeleine Guéritte. In Hofstetter, R., Riondet, X. Go, H.L. (à paraître, 2018). Itinéraires des pédagogues (titre provisoire). Presses Universitaires de Grenoble.

Jourdan, I. (2015). Posture, corps et voix de l'enseignant débutant :

Une démarche clinique de formation. Recherches \& éducations, 12, 2014, 105-116.

Kolly, B. (2018, à paraître). Anthropologie et mouvement Montessori : itinéraire d'une idée pédagogique. In R. Hofstetter, X. Riondet \& H.L. Go (à paraître, 2018). Itinéraires des pédagogues (titre provisoire). Presses Universitaires de Grenoble.

Kolly, B. (2015). Le statut de la norme chez Maria Montessori : « norme humaine » et norme sociale. In H.L. Go (dir). Normes pour apprendre. Nancy : Presses Universitaires de Nancy.

Marzano, M. (2009). Philosophie du corps. Paris : PUF.

Moulin, J.F. (2004). Le discours silencieux du corps enseignant. La communication non verbale du maître dans les pratiques de classe. Carrefours de l'éducation, 1/2004.

Pujade-Renaud, C. (1983). Le corps de l'enseignant dans la classe. Paris : ESF. 
Riondet, X. (2010). Le « corps » à l 'École dans la revue Les Cahiers pédagogiques : description foucaldienne et enjeux de la nouvelle configuration scolaire des Novateurs (1958-1989). Staps, vol. 89, no. 3, 2010, pp. 115-124.

Savoye, A. (2004). L'Education nouvelle en France, de son irrésistible ascension à son impossible pérennisation (1944-1970). In A. Ohayon, D. Ottavi, A. Savoye (2004). L'éducation nouvelle, histoire, présence et devenir. Bern : P. Lang, pp. 235-271.

Sensevy, G. (2014). Réponses aux recensions - à propos du Sens du savoir. Éducation et didactique, 8-1, 2014, 131-135.

Vigarello, G. (2004). Le corps redressé. Histoire d'un pouvoir pédagogique. Paris : A. Colin.

Vincent, G. (1994). L'éducation prisonnière de la forme scolaire? Scolarisation et socialisation dans les sociétés industrielles. Lyon : Presses Universitaires de Lyon.

Vincent, G. (1980). L'école primaire française. Etude sociologique. Lyon : Presses Universitaires de Lyon.

\section{NOTES}

1. Montessori précise : modifier, non créer. L'ambiance et le milieu n'est pas créateur ; la création est présentée comme interne à l'enfant.

2. Ce terme de «chair mentale " est étonnamment proche du terme proposé par Andrieu de « chair du cerveau ». Il faudrait ici approfondir une double lecture que nous faisons qu'esquisser.

3. Cette question serait à étudier précisément. Le rapport d'Alvarez à la pédagogie Montessori est en effet complexe : elle a bénéficié d'une formation Montessori, ses pratiques sont directement issues de la pédagogie, et certains des résultats qu'elle avance se retrouvent dans les ouvrages de la doctoresse. Pour autant, elle déclare continuer la recherche montessorienne, et à ce titre, se désolidarise du mouvement Montessori « orthodoxe », voire même, selon les périodes, de toute référence à Montessori.

4. Il existe aujourd'hui plusieurs lieux de formation à la pédagogie montessorienne. Les deux principaux centres sont l'Institut Supérieur Maria Montessori (seul habilité par l'A.M.I.) et le C.R.E.L.A.M. (Centre de Recherche, d'Etude et de Liaison des Activités Montessori), qui forme également des enseignants, propose des séminaires dans l'ouest de la France. Ces deux centres sont issus de personnes directement formées auprès de Maria Montessori. Nous pouvons mentionner, enfin, «La Source », à Manosque, qui forme des enseignants depuis les années 1980. Ce centre, plus informel, a notamment alimenté toute une vague de "petits pédagogues" montessoriens dans les années 1990.

5. Ce terme reprend l'expression d'Antoine Savoye, celui de "nébuleuse montessorienne». (Savoye, 2004, p. 235).

6. Voir Guttierez, L. \& Savoye, A., 2018 (à paraître)

7. Une thèse de doctorat en sociologie de l'éducation est en cours sur ces différents mouvements et leur apport dans l'enseignement classique, par Chrystel Huard, sous la direction de Bruno Robbes.

8. En prenant l'exemple du règlement de J.B. de La Salle, Foucault rappelle que le maître désigne les mouvements, en intercalant si besoin « un écolier léger et éventé soit entre deux qui soient sages et posés, un libertin seul ou entre deux qui ont de la piété » (cité p. 173).

9. Ces cartographies ainsi que quelques autres, sont également présentées dans un article à paraitre en 2019 dans la revue Recherche en éducation, intitulé «Abolir les frontières dans la classe $»$. 
10. Le fait qu'il soit aussi un garçon est fortuit. Etudier les mouvements des enfants en fonction du genre pourrait être une piste.

11. Ce dernier propose, en repartant de l'idée de Kuhn, d'imaginer une nouvelle forme scolaire à partir d'exemples existants, et les restituer et de les comprendre au mieux, afin, peut-être, d'en « organiser la croissance » (Sensevy, 2014)

\section{RÉSUMÉS}

Cet article entend tenter de rendre compte du régime spatio-temporel spécifique qui est celui des enfants de maternelle Montessori, ce dernier entrant en contradiction ou en friction avec la forme scolaire traditionnelle. Ces pratiques entendent en effet modifier le centre de gravité du « travail » de l'enseignant vers l'enfant, et pour cela libérer le mouvement de ce dernier.

This article attempts to give an account of the specific spatio-temporal mode of Montessori schools, which is in conflict with the traditional "school form". These practices intend to modify the center of gravity of the activities of the school, from the teacher towards the child, and for this to release the movement of the latter. We first propose to define what a Montessori practice might be, or to define the questions and problems that such an attempt at definition raises; we then seek to describe the primary effect that this spatio-temporal mode produces in the classroom: child walkers, or nomadic children.

INDEX

Mots-clés : Montessori, montessorisme, mouvement, enfants marcheurs, forme scolaire Abstract

Keywords : Montessori, Montessori pedagogy, movement, child walkers, school form

\section{AUTEUR}

\section{BÉRENGÈRE KOLLY}

UPEC-Espé de Créteil, Laboratoire Lettres, Idées, Savoirs 